\title{
La clasificación de las ciencias desde la filosofía del cierre categorial
}

David Alvargonzález

Universidad de Oviedo (España) 



\title{
La clasificación de las ciencias desde la filosofía del cierre categorial
}

\section{The classification of sciences from the categorial closure phi- losophy}

\author{
David Alvargonzález \\ Universidad de Oviedo (España) \\ dalvar@uniovi.es
}

Fecha de recepción: 1 de febrero de 2018

Fecha de aceptación: 5 de mayo de 2019

\section{Resumen}

En este artículo se presenta una clasificación de las ciencias realizada desde la filosofía del cierre categorial de Gustavo Bueno. En primer lugar, voy a comentar ciertos presupuestos de la teoría buenista que afectan a esta tarea clasificatoria. Luego distinguiré las clasificaciones basadas en criterios ontológicos de las basadas en criterios gnoseológicos. A continuación, presentaré un criterio gnoseológico de clasificación de las ciencias que, aunque es diferente del propuesto por Gustavo Bueno, es interno a la filosofía del cierre categorial. Pasaré luego a aplicar ese criterio y propondré una clasificación de las ciencias que trataré de justificar confrontándola con otras alternativas.

Palabras clave: Gustavo Bueno; Clasificación de las ciencias; Teoría del cierre categorial; Ciencias formales; Ciencias humanas; Ciencias naturales; Ciencias históricas; Ciencias etológicas

\footnotetext{
Abstract

This paper presents a classification of the sciences carried out from the Gustavo Bueno's philosophy of categorial closure. Firstly, I will comment on certain assumptions of Bueno's theory that affect this classificatory task. Then, I will distinguish the classifications based on ontological criteria from those based on gnoseological criteria. Next, I will present a gnoseological criterion of classification of sciences that, although different from that proposed by Gustavo Bueno, is internal to the philosophy of categorial closure. Finally, I will apply that criterion and I will propose a classification of the sciences, confronting it with other alternatives.

Keywords: Gustavo Bueno; Classification of sciences; Categorial closure; Formal sciences; Human sciences; Natural sciences; Historical sciences; Ethological sciences
} 


\section{INTRODUCGIÓN}

La clasificación de las ciencias es una tarea típica de la filosofía de la ciencia desde la aparición de la multiplicidad de las ciencias modernas, tras la revolución científica y tecnológica. Gustavo Bueno, en su teoría del cierre categorial, propuso la clasificación de las ciencias en dos grupos: ciencias naturales y ciencias humanas (y etológicas) (Bueno 1976 y 1993). David Alvargonzález discutió esa teoría en un trabajo que fue contestado por Gustavo Bueno (Alvargonzález 1989; Bueno 1989), y estudió ciertos problemas específicos de la teoría de juegos y de las ciencias divinas de la escolástica española tardía que resultaban relevantes en aquella discusión (Alvargonzález 1994 y 2011). Partiendo de esa polémica, en este artículo se trata de proponer una teoría alternativa a la de Gustavo Bueno que, sin embargo, sea compatible con los presupuestos de su teoría del cierre categorial. Para ello se analizan las diferencias y las semejanzas entre las ciencias y se propone una clasificación en cuatro grupos: formales, naturales, humanas (y etológicas), e históricas. Se explican los fundamentos de esta clasificación, y se compara con otras clasificaciones alternativas que han sido propuestas en los dos últimos siglos.

\section{ALGUNOS PRESUPUESTOS DE LA FILOSOFÍA DE GUSTAVO BUENO}

Gustavo Bueno, ya desde sus primeras obras, distinguió entre los saberes de primer grado y los saberes de segundo grado. Las técnicas, las tecnologías, las artes, y algunas disciplinas prudenciales tales como la ética, la política y la jurisprudencia son conocimientos de primer grado. Las ciencias modernas, la física, la química, la biología, las matemáticas, la psicología, la lingüística, entre otras, también pueden considerarse conocimientos de primer grado acerca de ciertas regiones del mundo. Los conocimientos de primer grado construyen conceptos: así existen conceptos científicos (entropía, energía cinética, circunferencia, célula), técnicos (dintel, arquitrabe, quilla, bronce), tecnológicos (tomografía, electroencefalograma, dron, GPS), artísticos (soneto, capitel, escala, sfumato, contrapposto), jurídicos (pena, sentencia, habeas corpus), políticos (partido, circunscripción, consenso, coalición), etc. La filosofía académica, sin embargo, es un conocimiento de segundo grado que implica la existencia previa de los saberes de primer grado y de sus conceptos. La filosofía construye ideas que implican tomar en consideración una amplia variedad de conocimientos de primer grado y de conceptos. Causalidad, verdad, belleza, bien, identidad, cultura, hombre, persona, libertad, justicia son ideas (Bueno 1970, 1995a, 2013).

De acuerdo con esta distinción, la clasificación de las ciencias implica clasificar saberes de primer grado y conduce a grupos tales como ciencias formales, naturales, humanas, idiográficas, nomotéticas, empíricas, etc. La clasificación de las disciplinas filosóficas es una tarea completamente diferente y da como resultado grupos que 


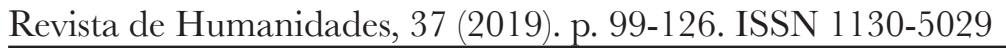

cobran significado en el contexto de un sistema filosófico: disciplinas ontológicas, antropológicas, epistemológicas, de estética, de filosofía ética y política, etc. (Bueno 2004).

En segundo lugar, la filosofía de Gustavo Bueno supone un pluralismo tanto ontológico como gnoseológico que se opone a todo tipo de monismo, en la línea de ciertos filósofos de la Escuela de Stanford (Dupré 1993, Galison y Stump 1996, Cartwright 1999). Por lo que aquí nos afecta, se opone al monismo del orden, lo cual implica que no existe un "sistema de las ciencias", un único sistema científico en el que los teoremas de todas las ciencias queden coordinados por los mismos principios. Las ciencias son sistemas, pero no existe un sistema de las ciencias ya que, aunque ciertas ciencias puedan compartir algunos procedimientos metodológicos con otras, no hay un conjunto de principios que sea común a todas las ciencias y que coordine los teoremas de todas las ciencias. Lo mismo que un grupo de Estados políticos no es automáticamente un Estado, y lo mismo que un conjunto de círculos no da lugar a un círculo, un conjunto de sistemas no siempre da lugar a un sistema.

La teoría del cierre categorial mantiene una cautela y una actitud expectante ante los proyectos de unificación de las ciencias. Como es sabido, en el siglo pasado, Rudolf Carnap y Otto Neurath, miembros del Círculo de Viena, promovieron la unificación de las ciencias por medio de un lenguaje unificado con el objetivo de construir una visión científica del mundo, un monismo sin metafísica (Neurath et al. 1938). Desde entonces ha habido más propuestas en favor de la unificación del conocimiento, como las de John Brockman y Edward O. Wilson (Brockman 1995; Wilson 1998) por no hablar de los proyectos transdisciplinarios (Alvargonzález 2011). El proyecto de unificación de las ciencias del Círculo de Viena fue abandonado por sus propios promotores por inviable y, en cuanto a los proyectos actualmente en curso, no se puede afirmar que la unidad de las ciencias se haya conseguido o esté en vías de conseguirse.

\section{DIFERENCIA ENTRE LAS CLASIFICACIONES ONTOLÓGICAS Y LAS GNOSEOLÓGICAS}

La tendencia a plantear el problema de la clasificación de las ciencias como un problema ontológico ha sido constante en la historia de la filosofía. Se trata de suponer que las ciencias describen el mundo o se adecuan a la realidad como un mapa al terreno y, por tanto, la clasificación de las ciencias remite, en último término, a la división de la realidad en partes. Esta confusión entre el problema gnoseológico de la clasificación de las ciencias y el problema ontológico de determinar las partes de la realidad entendida como una totalidad atributiva se aprecia claramente en las clasificaciones de las ciencias inspiradas en el idealismo hegeliano. Hegel, en su Enciclopedia de las ciencias filosóficas, ordenó su sistema ontológico por medio de la conocida tripartición entre la lógica, la filosofía de la naturaleza y la filosofía 
del espíritu (Hegel 1817). Esta distinción ontológica tuvo una gran influencia desde entonces. Engels, en la Dialéctica de la Naturaleza, también dividió las ciencias en tres grupos: las ciencias del pensamiento (que se pueden coordinar con la lógica), las ciencias naturales y las ciencias humanas (Engels, 1876). Más tarde, Wilhelm Wundt distinguió, por una parte, las ciencias reales de las formales (la lógica y las matemáticas) y, entre las reales, diferenció las ciencias naturales de las ciencias del espíritu (Wundt 1889). En la misma línea, Wilhelm Ostwald habló de unas ciencias del orden (lógica, matemáticas y mecánica), unas ciencias energéticas (física, química, fisiología), y unas ciencias biológicas (psicología, culturología y sociología) (Ostwald 1914). Siguiendo los pasos de Engels y Lenin, Boniface Kedrov estableció la doctrina del Diamat haciendo uso de un esquema ontológico trinitario: las ciencias naturales, las ciencias sociales y las ciencias filosóficas entre las que incluyó la lógica (Kedrov 1961). Kedrov argumenta que "las ciencias deben disponerse en una sucesión lógica y en cierta forma enlazarse entre sí, no porque eso nos parezca cómodo, sino porque así están enlazados ente sí los objetos mismos, las formas mismas del movimiento de la materia, estudiados y reflejados por las ciencias correspondientes" (Kedrov 1961, 28). Desde presupuestos filosóficos muy diferentes, Mario Bunge también propuso una clasificación de las ciencias de acuerdo con su objeto de estudio y fue a dar con la misma estructura que hubiera propuesto Wundt: la distinción de ciencias formales frente a las factuales, y la división de las factuales en dos grupos: naturales y culturales.

En este artículo supondré que la tarea gnoseológica de clasificar las ciencias de acuerdo con su estructura y su funcionamiento puede distinguirse de la tarea ontológica de determinar las partes de la realidad considerada como una totalidad. La teoría del cierre categorial se construye en parte como una crítica a las concepciones descripcionistas, teoreticistas y adecuacionistas de la verdad científica, porque niega que ésta sea una descripción de la realidad, una mera elaboración teórica o un mapa del mundo. Las ciencias no tienen un único objeto de estudio (la naturaleza, la cultura, la vida, etc.) sino un campo complejo constituido por una multitud de objetos. (Bueno 1993).

\section{PROPUESTA DE UNA CLASIFICACIÓN GNOSEOLÓGICA DE LAS GIENCIAS}

Como he dicho, la idea de ciencia del cierre categorial de Gustavo Bueno implica la negación del monismo gnoseológico, y el reconocimiento de la multiplicidad de las ciencias y de su mutua irreductibilidad. Esto significa que partimos de una pluralidad de ciencias, y que no existe una única ciencia considerada como un todo que incluya a las ciencias realmente existentes como partes suyas. Las ciencias que voy a tomar en consideración son las ciencias en sentido moderno, incluyendo la geometría que puede considerarse retrospectivamente como una ciencia estricta. También incluiré 
Revista de Humanidades, 37 (2019). p. 99-126. ISSN 1130-5029

las ciencias en sentido ampliado, las llamadas ciencias humanas o sociales, incluyendo la historia. La vía de clasificación que voy a proponer es ascendente, por tipificación con lo cual la clasificación propuesta se acerca mucho a una tipología. Ahora bien, la vía tipológica, ascendente, se complementa con el establecimiento de unas partes determinantes comunes a todas las ciencias que resultan ser claves a la hora de clasificarlas, de modo que se completa con un regreso descendente que, una vez conseguido, permitiría entender la clasificación como una taxonomía. La pluralidad de ciencias no es un caos, y permite establecer una clasificación de las ciencias basada en su propia estructura interna en la medida en que ésta afecta a su propia cientificidad.

De acuerdo con la filosofía materialista, las ciencias, las técnicas y las tecnologías son actividades de carácter operatorio manual ya que implican la realización de operaciones y transformaciones sobre cuerpos, pero mientras las técnicas y las tecnologías deben estar orientadas a fines prácticos, las ciencias no implican necesariamente este requerimiento. La organización interna de una determinada ciencia no depende del logro de ningún propósito particular (como ocurre en las técnicas y las tecnologías) sino que depende de sus teoremas que son verdades apodícticas universales, y de sus principios que coordinan los teoremas y hacen posible el cierre categorial.

La clasificación de las técnicas y de las tecnologías puede fundamentarse en los objetivos prácticos que persiguen: por ejemplo, así se pueden distinguir con claridad las tecnologías médicas de las aeroespaciales. Sin embargo, este criterio práctico de clasificación no es de utilidad cuando nos referimos a las ciencias ya que su desarrollo no depende del logro de un determinado objetivo particular.

Con todo, de acuerdo con los presupuestos materialistas, las ciencias son actividades prácticas que suponen la intervención, transformación y constitución de partes importantes de nuestro mundo actual, y son el procedimiento para alcanzar verdades universales, contenidas en sus teoremas y sus principios. Esto nos proporciona indicios para buscar ciertos criterios de clasificación de las ciencias fijándonos en su funcionamiento: nos interesa especialmente el modo en que los científicos operan y los diferentes tipos de cuerpos con los que operan, así como los procedimientos que conducen al establecimiento de los teoremas y principios universales. Formulado de la manera más general posible el criterio de clasificación que voy a utilizar es el siguiente: puesto que una ciencia implica siempre un conjunto de operaciones sobre los términos de su campo, los materiales sobre los que se opera en cada caso, y el modo de operarlos, afectan a la propia estructura de la ciencia, y ello da lugar a las diferentes maneras de darse la ciencia. Defenderé que este criterio es interno a las ciencias desde el momento en que está tomado de la estructura interna de sus campos y del grado de cientificidad que alcanzan esos campos en cada caso.

1. En las ciencias llamadas "formales" (la lógica y las matemáticas) los científicos operan con ciertos objetos sui generis, objetos tipográficos, que son signos auto-referentes. 
2. En las ciencias llamadas "naturales" (la física, la química, la biología, la geología, etc.) los científicos operan con objetos que no son signos: cuerpos en movimiento, sustancias, compuestos, organismos, rocas, etc. En estas ciencias también encontramos signos, pero estos son usados para referirse a los objetos, no son auto-referentes como en el caso de las ciencias formales. Los objetos con los que se opera en las ciencias naturales son objetos a los que no se les reconocen operaciones. En el caso en el que algunos de esos objetos sean organismos que realizan operaciones, esas operaciones no son tenidas en cuenta formalmente.

3. En las ciencias humanas y etológicas (lingüística, antropología cultural, sociología, psicología, etología, etc.) los científicos operan con objetos inertes, pero también operan sobre las propias operaciones de los sujetos temáticos (hablantes, nativos, agentes sociales, animales, etc.). Cuando esos sujetos temáticos son animales, estamos en los campos de la psicología y la sociología animal, y de la etología; cuando son sujetos humanos dotados de un lenguaje de palabras similar al del científico, estamos en los campos de las ciencias humanas. Los campos de las ciencias humanas y etológicas tienen dos niveles de operaciones: las operaciones del científico y las operaciones de los sujetos a quienes se estudia (ya sean animales o humanos).

4. En las ciencias históricas, los científicos operan con un conjunto de objetos: vestigios, monumentos, ruinas, documentos. Estos objetos de etiología humana no podrían distinguirse de su entorno si no se supusieran las operaciones de los individuos pretéritos que los han construido. Por tanto, aunque el sujeto temático no está directamente en el campo, pues está muerto, hace falta reconstruir sus operaciones para que esos objetos del campo adquieran sentido. En la historia esos objetos incluyen textos de un lenguaje de palabras (narraciones, informes, documentos) mientras que en la prehistoria y la arqueología los vestigios no incluyen lenguajes escritos.

Así pues, dependiendo de la naturaleza de los términos operados se propone distinguir cuatro grupos de ciencias: "formales", "naturales", humanas y etológicas y, por último, históricas. (ver Tabla I).

Esta propuesta no es nueva en cuanto a sus resultados pues en 1893 André Lalande ya elaboró una clasificación muy parecida. Dice Lalande:

Del examen de todas las clasificaciones existentes de las ciencias resultan cuatro grandes divisiones reconocidas por todos: primera, ciencias matemáticas; segunda, ciencias físicas y naturales, que tienen por objeto descubrir leyes del mundo material, orgánico e inorgánico; tercera, ciencias morales, que se ocupan de los fenómenos y las leyes del mundo espiritual $[\ldots]$ y, finalmente, cuarta, ciencias de la erudición, cuyo objeto lo forman no las leyes, sino los hechos, y la historia es su ejemplo más considerable. En el orden ahora enumerado, las ciencias pasan de los objetos más abstractos a los más concretos, de lo inteligible a lo sensible, de la razón a la experiencia (Lalande 1893, 60-61). 
Tabla I. La clasificación de las ciencias

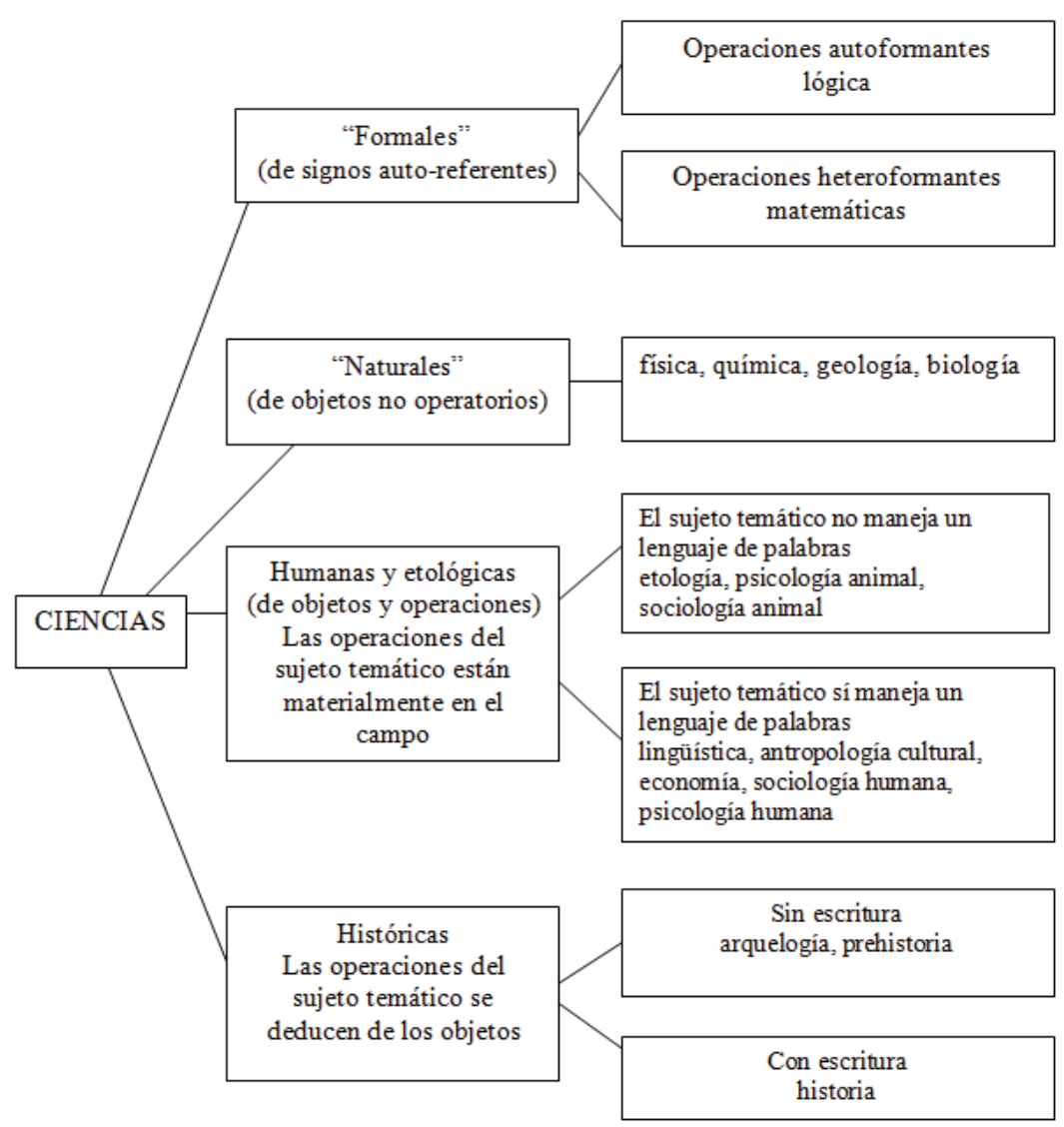

Pero la cuestión radica en discutir sus fundamentos ya que los propuestos por Lalande parecen muy frágiles. En lo que sigue, mantendré que los fundamentos de esta clasificación se pueden buscar en la estructura operatoria de los diferentes campos científicos en la medida en que afecta a su grado de cientificidad.

Las etiquetas usadas para designar a estos cuatro grupos de ciencias (ciencias formales, naturales, humanas y etológicas, e históricas) son todas ellas bastante desafortunadas y no ayudan a clarificar los fundamentos de la clasificación. Desde los presupuestos de este trabajo los rótulos de ciencias formales frente a ciencias reales o empíricas inducen a confusión ya que no hay una ciencia de formas puras y además las ciencias formales también son ciencias reales y empíricas. Las etiquetas de ciencias de la naturaleza frente a ciencias humanas o ciencias de la cultura también son desafortunadas porque la Naturaleza no existe como tal sin el hombre, y porque el hombre y su cultura también tienen muchos rasgos que son producto de la naturaleza. La distinción entre ciencias humanas e históricas también es oscura 
ya que las ciencias históricas son, desde luego, ciencias que tratan de los hombres y de sus productos. A pesar de estos inconvenientes, no he querido proponer nombres nuevos para estos grupos de ciencias ya que el uso de las etiquetas actuales está ya tan arraigado que me parece imposible cambiarlo.

\section{JUSTIFICACIÓN DE LA CLASIFICACIÓN PROPUESTA}

\subsection{Sobre la distinción entre las ciencias formales y el resto de las ciencias}

En el siglo XIX, Wilhelm Wundt introdujo la expresión "ciencias formales" para referirse a un grupo de ciencias que, según él, no se interesarían por la realidad empírica, sino que tratarían de objetos puramente ideales y estudiarían sus propiedades formales (Wundt 1889). Frente a esas ciencias estarían las ciencias "reales", "experimentales" o "empíricas", que incluirían las ciencias de la Naturaleza y las ciencias del Espíritu. Wundt subrayó la especificidad de las ciencias formales, pero no fue capaz de dar con sus rasgos distintivos ya que tuvo que suponer que existían unas formas puras, separadas de la materia (algo parecido a los ángeles o los espíritus incorpóreos tal como los entendió Suárez) que serían los objetos de estudio de esas ciencias. En todo caso, es necesario recordar que las ciencias que él llamo reales o empíricas también estudian muchas formas que están encarnadas en el mundo real: órbitas, trayectorias, morfologías, estructuras, etc.

Como se ha dicho ya, la filosofía del cierre categorial supone que toda ciencia es empírica y tiene un campo de objetos fisicalistas operables de modo que es imposible que una ciencia se constituya exclusivamente en torno a entes ideales o formas puras, ya que se niega la existencia de formas separadas de la materia. En el caso de las ciencias formales esos objetos operados por los científicos son las propias materialidades tipográficas. Esos signos-objeto son auto-referentes ya que, desde un punto de vista matemático (o lógico), las eventuales referencias que puedan tener fuera del campo de esa ciencia no son directamente pertinentes en el momento de probar los teoremas. El resto de las ciencias (naturales, humanas, etológicas, históricas) también utilizan signos, pero sus signos siempre van referidos a objetos que no son signos: en las ciencias no formales se puede distinguir un estrato proposicional y un estrato objetual ya que ambos estratos no coinciden. La geometría de Euclides suele considerarse la primera ciencia formal. En ella, a partir de operaciones y relaciones con signos-objeto auto-referentes, construidos con regla y compás, y dotados de materialidad tipográfica, los geómetras griegos construyeron multitud de teoremas y los coordinaron entre sí por medio de principios, axiomas y nociones comunes.

En un primer momento, podría parecer que la distinción entre ciencias formales y no formales es superficial, no gnoseológica, ya que todas ellas son ciencias reales y empíricas, y la circunstancia de que los campos de las ciencias formales estén hechos con objetos auto-referentes no afecta para nada a su grado de cientificidad pues las ciencias formales son ciencias estrictas. 
Revista de Humanidades, 37 (2019). p. 99-126. ISSN 1130-5029

Sin embargo, hay al menos dos sentidos en los que el carácter auto-referente de los términos de estas ciencias determina su estructura gnoseológica.

En primer lugar, me refiero al hecho de que las matemáticas, a diferencia de otras ciencias no formales, se dicen en plural porque no existe un sistema unificado de todas las disciplinas matemáticas que esté coordinado por unos mismos principios comunes. Las disciplinas matemáticas son múltiples (geometría, aritmética, álgebra, análisis, cálculo, topología, etc.) y cada una de ellas, como ocurre en otras ciencias estrictas, tiene un campo formado por un conjunto de teoremas universalmente verdaderos: los teoremas son sistemas cuyas partes están coordinadas por leyes $\mathrm{y}$, a su vez, los teoremas están coordinados entre sí por medio de principios para asegurar el cierre categorial. Gustavo Bueno ha reconocido en diversas ocasiones esta anomalía del campo de las matemáticas que podemos formular como la "irreductibilidad mutua de las disciplinas matemáticas", y ha argumentado en contra de la concepción monista del campo de las matemáticas y contra la idea de que exista una "categoría matemática primordial" de la que el resto fueran modulaciones o transformaciones (Bueno 1993, 268-89; Bueno 2000a, 70-71). Pues bien, sostengo que esta irreductibilidad mutua de las disciplinas matemáticas es consecuencia del carácter auto-referente de sus construcciones que permite un desarrollo de los términos y las operaciones en direcciones muy diferentes e incompatibles entre sí. Por ejemplo, la geometría de Euclides define la equivalencia entre dos configuraciones de su campo por medio de transformaciones isométricas (rotaciones, traslaciones, reflexiones, etc.) en las que se conservan las medidas de las longitudes, de los ángulos, de las áreas, etc. Por su parte, en el campo de la topología dos configuraciones son equivalentes si tienen el mismo número de trozos, huecos o intersecciones, y las longitudes, los ángulos, las áreas y los volúmenes se pueden estirar, encoger, doblar y retorcer siempre que no se rompan ni separen las partes que estaban unidas. Partiendo de principios de equivalencia tan dispares es lógico que los teoremas geométricos y topológicos sigan cursos y ritmos diferentes, cuando no incompatibles. Y esto sin perjuicio de que puedan abrirse espacios para la interdisciplinariedad: pero esas relaciones interdisciplinares, como también ocurre con las ciencias no formales (la bioquímica, la geofísica, la neuropsicología, etc.), implican la independencia y soberanía de las disciplinas involucradas, y su mutua irreductibilidad (Alvargonzález 2011). En todo caso, no hay duda alguna de que la geometría o la topología sean ciencias estrictas con sus teoremas universales bien establecidos y sus principios específicos de coordinación de los teoremas. Consideraciones parecidas podrían hacerse a propósito de las disciplinas lógicas, por ejemplo, de la lógica de clases frente a la lógica de relaciones. En las ciencias no formales, en especial en las ciencias llamadas "naturales", no ocurre esta proliferación de disciplinas científicas: la mecánica de Newton logró que todos los teoremas físicos se coordinaran bajo los mismos tres principios; la química clásica se constituyó como un campo único, denotado por los elementos de la tabla periódica, con unos únicos principios (los principios de Lavoisier, Dalton y Proust). 
En segundo lugar, la distinción entre "ciencias formales" y "ciencias reales" tiene significado gnoseológico porque las operaciones de las ciencias no formales están semánticamente determinadas por las propias materialidades de los objetos operados mientras que el formalismo de las matemáticas y de la lógica permite cierta desconexión semántica de modo que las operaciones de estas ciencias se dotan de contenido por vía pragmática y sintáctica. Esto implica que, en las ciencias formales los motivos por los que unos términos se componen operatoriamente con otros, y los mismos resultados de las operaciones, obedecen a una estrategia pragmática, exterior a los términos operados, mientras que en las ciencias reales esos motivos y esos resultados tienen que ver con el contenido material mismo de los objetos operados (por ejemplo, las masas que efectivamente se atraen de acuerdo a la ley de la gravitación). Así, en las ciencias formales los principios que regulan las operaciones serían pragmáticamente rectos y semánticamente oblicuos (externos), mientras que en las ciencias reales sería al revés ya que la semántica de los objetos operados tendría una influencia "recta" mientras los aspectos pragmáticos son "oblicuos" (Bueno 1976, 480-484). Esta circunstancia afecta al modo de darse la verdad científica en las ciencias formales. Desde luego, la teoría de la verdad científica entendida como una identidad sintética se aplica por igual a ambos grupos de ciencias, pero en las ciencias formales, debido al carácter auto-referente de su campo, la verdad científica se aproxima mucho a la verdad teoreticista, a la verdad sintáctica entendida como coherencia. Esto es así porque, en las ciencias formales, las construcciones proposicionales coinciden con las construcciones objetuales, y el cierre objetual coincide con el cierre proposicional. Esto no ocurre en el resto de las ciencias (Bueno 1992, 126-28).

Por último, desde la filosofía del cierre categorial, las ciencias formales se dividen en dos grupos: lógica y matemáticas de acuerdo con el siguiente criterio de demarcación: la lógica se caracterizaría por el uso de operaciones autoformantes en las que el resultado de la operación es el mismo término de partida (operaciones como $\mathrm{A}^{*} \mathrm{~A}=\mathrm{A} ; \mathrm{p} \wedge \mathrm{p} \leftrightarrow \mathrm{p} ; \mathrm{p} \vee \mathrm{p} \leftrightarrow \mathrm{p} ; \neg \neg \mathrm{p} \leftrightarrow \mathrm{p}$ ); en las matemáticas, sin embargo, se usan preferentemente operaciones heteroformantes en las cuales, al operar un término consigo mismo, aparecen términos diferentes (operaciones del tipo $\mathrm{A} * \mathrm{~A} \neq \mathrm{A} ; \mathrm{a}+\mathrm{a}=$ $2 \mathrm{a} ; \mathrm{a}-\mathrm{a}=0 ; \mathrm{a} * \mathrm{a}=\mathrm{a}^{2} ; \mathrm{a} / \mathrm{a}=1$ ) (Bueno 1978, 1979). Esta propuesta de demarcación es internamente gnoseológica, y se articula con el proyecto clasificatorio defendido aquí ya que toma como criterio la naturaleza de las operaciones realizadas por el científico.

5.2. Sobre la distinción entre ciencias naturales y ciencias humanas y etológicas

Como ha quedado explicado en el apartado segundo, la concepción de la ciencia como mera representación de la realidad hizo que el problema de la clasificación de 


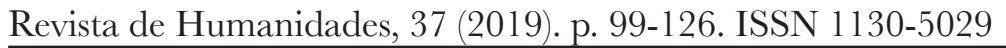

las ciencias quedase resuelto por referencia a las dos grandes esferas ontológicas que, de acuerdo con la filosofía idealista alemana, componían la realidad, la Naturaleza y la Cultura, de modo que cada uno de esos ámbitos ontológicos debía ser estudiado por un grupo específico de ciencias, las ciencias naturales y las ciencias de la cultura (también llamadas ciencias morales, ciencias sociales, ciencias del espíritu, o ciencias humanas). No es este el lugar de discutir la pertinencia del dualismo ontológico del idealismo alemán, pero, para mis propósitos actuales, bastará con decir que la existencia de las ciencias realmente existentes plantea dificultades importantes a la dicotomía Naturaleza / Cultura: los contenidos de las ciencias formales ¿son naturales o culturales? Las llamadas "culturas animales" ¿son culturales o naturales? ¿Acaso las ciencias naturales no incluyen en sus campos gran cantidad de contenidos culturales, empezando por todos los aparatos científicos?

Esta fundamentación ontológica de esas dos clases de ciencias era además compatible con la idea de que las ciencias humanas, debido a su objeto de estudio (la humanidad, la cultura, la sociedad, etc.) hacían uso de unos métodos especiales. Dilthey y Simmel defendieron la famosa distinción entre el procedimiento mecánico de la "explicación", propio de las ciencias naturales, y el procedimiento empático de la "comprensión", típico de las ciencias humanas (Dilthey 1883; Simmel 1920). Pero la existencia de dos metodologías radicalmente diferentes, explicación y compresión, que se corresponderían con dos diferentes tipos de ciencias, plantea el problema de hasta qué punto este dualismo metodológico es compatible con la unidad de la idea de ciencia y con la unidad de la idea de verdad científica. Parece que la expresión "verdad científica" no significaría lo mismo cuando se refiere a una "explicación" construida en las ciencias naturales o formales que cuando se refiere a la "compresión" de un acontecimiento histórico.

Gustavo Bueno también defendió la pertinencia de la división de las ciencias en dos grupos, naturales y humanas, pero fundamentó esta distinción en la diferente estructura gnoseológica de las ciencias. La diferencia entre esos dos grupos de ciencias habría que ponerla en la naturaleza de los términos operados por los científicos: en el caso de las ciencias naturales, los términos operados son objetos inertes o considerados como inertes, mientras que en los campos de las ciencias humanas aparecen las operaciones de los sujetos temáticos: hablantes, nativos, agentes sociales y económicos, animales, etc. Bueno defendió que los campos de las ciencias humanas y etológicas tienen dos niveles operatorios distintos: el nivel de operaciones de los científicos y el nivel de las operaciones de los sujetos a los que se estudia ya que esas operaciones son tomadas como objeto de estudio. Precisamente las ciencias humanas y etológicas tratarían de dar cuenta de las operaciones de esos sujetos temáticos conectándolas con otros términos del campo. Esta definición de las ciencias humanas implica que la psicología y la sociología animal y la etología también deben clasificarse junto con el resto de las ciencias humanas ya que ciertos sujetos animales también realizan operaciones análogas a las humanas. Esta proximidad entre las ciencias humanas y las ciencias etológicas no es más que una 
consecuencia de la proximidad operatoria, conductual entre los hombres y ciertos animales.

La circunstancia de que en los campos de las ciencias humanas figuren como términos las operaciones de otros sujetos humanos o animales tiene un profundo significado gnoseológico porque afecta a la propia posibilidad de construir teoremas científicos universalmente válidos. Según la filosofía del cierre categorial, el proceso de construcción de las verdades científicas parte de las operaciones de los científicos con los términos del campo para determinar ciertas regularidades en esas operaciones y en las relaciones que surgen de ellas. La verdad científica se consigue cuando los aspectos subjetivos de las diferentes operaciones quedan neutralizados unos con otros, y se logran establecer relaciones entre términos que son independientes de los científicos. Por ejemplo, las leyes de Kepler neutralizan los aspectos subjetivos, ligados a un espacio y un tiempo particular, de multitud de observaciones astronómicas del Sol, la Luna y los planetas (originariamente Marte) de modo que las relaciones entre los términos que se establecen en las tres leyes son independientes de los científicos y de las observaciones concretas que posibilitaron su construcción. En los campos de las ciencias humanas y etológicas, sin embargo, las operaciones de los sujetos temáticos (hombres o animales) tienen que estar presentes como términos ya que si no lo estuvieran esas ciencias se convertirían en ciencias formales o naturales y esta circunstancia dificulta la posibilidad de construir verdades "objetivas" ya que la objetividad exige la neutralización del sujeto. Cuanto mayor sea el grado de eliminación de las operaciones, tanto más objetiva y científica será la disciplina, pero, al mismo tiempo, su estatus de ciencia "humana" será puesto en peligro. Por el contrario, si las operaciones de los sujetos temáticos son mantenidas en el campo, entonces quedará claro que estamos en una ciencia del grupo de las ciencias humanas y etológicas, pero la objetividad de los teoremas construidos en esa disciplina será problemática. (Bueno 1978 y 2013).

\subsection{La especificidad de las ciencias históricas}

En las ciencias humanas y etológicas los científicos interactúan en mayor o menor medida con los sujetos temáticos (animales y humanos) cuyas operaciones son reconocidas comotales enel campo. Losteoremas de esas cienciastratarán precisamente de explicar esas operaciones. En las ciencias históricas, sin embargo, los agentes históricos(César,FelipeII,Napoleón)yaestánmuertosytansólonosquedanlosvestigios y los documentos. A partir de esos objetos, las operaciones de los sujetos temáticos pretéritos tienen que ser supuestas, inferidas (otros preferirán decir "abducidas"), de modo que el historiador puebla esas reliquias con los fantasmas de los muertos.

Hay tres indicios muy fuertes que nos permiten suponer que la distinción propuesta entre las ciencias históricas y el resto de ciencias es una distinción interna gnoseológica 
En primer lugar, me voy a referir a la diferencia entre los cierres fijos, propios de las ciencias históricas, y los cierres flotantes, propios del resto de las ciencias, tal como la expuso Gustavo Bueno (Bueno 1976, 815-842; 1980, 63-64). Reelaborando la distinción de Bueno puede decirse que, en la historia, las operaciones pretéritas supuestas resultan, a cierta escala, irrepetibles ya que no se puede experimentar con los acontecimientos pasados de la manera como se hace en las ciencias naturales en las que se repiten los experimentos (por ejemplo, las reacciones químicas) o como también se hace en el resto de las ciencias humanas en las que los sujetos temáticos están presentes y se puede interactuar con ellos repetidamente. La posibilidad de repetir los procesos es una característica constitutiva esencial de toda operación ya que es lo que hace posible que los resultados de la operación se puedan desconectar de los términos operados. Recordemos que las operaciones se diferencian de las relaciones precisamente porque en las relaciones a partir de términos se obtiene una totalidad (por ejemplo, la igualdad $\mathrm{a}=\mathrm{b}$, en masa, dada como totalidad en el equilibrio de los platillos de una balanza), mientras que, en las operaciones, a partir de unos términos se obtienen otros que pueden desconectarse de los términos operados, de modo que el camino de vuelta, desde los resultados a los términos originales operados, no se deduce del resultado de la operación (por ejemplo, en las operaciones básicas de la aritmética). Por tanto, en las ciencias históricas las operaciones pretéritas quedan "fijas" mientras que en el resto de las ciencias los términos operados quedan liberados, quedan "flotando" listos para ser operados con otros términos.

Según Bueno, la distinción universal / particular, en el uso que se hace para diferenciar las ciencias nomotéticas de las idiográficas, no es pertinente cuando se aplica a los términos de los campos respectivos ya que ambos tipos de ciencias tienen términos particulares y universales. La diferencia entre las ciencias históricas y el resto de las ciencias reside en que en la historia aparecen configuraciones particulares a la escala de los resultados globales, configuraciones tales como puedan ser "imperio romano", "iglesia católica" o "revolución de octubre". Son configuraciones singulares porque la expansión histórica de los estados va dando lugar a una totalidad que tiene una estructura atributiva y cuyas partes están ligadas por contigüidad espacial y temporal. Las ciencias no históricas están privadas de esta singularidad idiográfica global. Es este individuo global el que es único y con el cual no cabe la posibilidad de repetición propia de la experimentación operatoria en el resto de las ciencias. Gustavo Bueno, dentro de las variedades de la verdad en sentido lógico-material, distinguió la verdad de las ciencias no históricas frente a la verdad idiográfica que él llamó factual (Bueno 2000b, 273-305). Sólo en un sentido muy débil, vago y problemático podrían aplicarse de un modo distributivo unas situaciones históricas a otras, como cuando se intenta arrojar cierta luz sobre la revolución de octubre apelando a lo ocurrido en la convención termidoriana de la revolución francesa.

Un segundo indicio que nos muestra la relevancia gnoseológica de la especificidad de las ciencias históricas es que, dado que éstas tienen que reconstruir las operaciones de los sujetos pretéritos, unas operaciones de etiología humana, la 
teoría de la verdad científica por adecuación o correspondencia resulta cercana a su modo de proceder. De acuerdo con la doctrina del verum est factum que Giambattista Vico reivindicó en su De Antiquissima, los hombres sólo pueden llegar a conocer realmente las causas de las cosas que ellos mismos han hecho, y el resto de las causas (diríamos nosotros, el ámbito de las ciencias naturales) sólo las conoce Dios padre, que es el sumo hacedor, de modo que los hombres tenemos que conformarnos con un conocimiento parcial (Vico 1710, segunda respuesta, IV). Thomas Hobbes habría expuesto una doctrina similar en su De homine: la geometría, la política y la ética, en cuanto hechas por los hombres, pueden ser conocidas a priori, mientras que sólo podemos tener un conocimiento parcial y conjeturar las causas de los procesos naturales, hechos por Dios, ya que un mismo efecto puede lograrse de diferentes modos (Hobbes 1658; Mondolfo 1969) Desde la idea de verdad científica de la filosofía del cierre categorial, en las ciencias históricas hay una reconstrucción de unas operaciones que existieron en otro tiempo y que se suponen análogas a las de los historiadores actuales. Esa reconstrucción es posible gracias a la identidad sintética urdida en una malla espacio temporal y causal en la que se sitúan los vestigios y los documentos. Sólo que aquí sí hay unos sujetos operatorios pretéritos que efectivamente desplegaron sus operaciones, mientras que, en las ciencias naturales, dado que el materialismo niega la posibilidad del Dios pantocrátor, la verdad científica no puede entenderse como adecuación con las operaciones de nadie.

Un tercer indicio de la relevancia gnoseológica de distinguir las ciencias históricas del resto de las ciencias humanas es el siguiente: las ciencias humanas no históricas permiten operar con los términos del campo en las mismas circunstancias que se dan en el resto de las ciencias ya que las operaciones del científico sobre el sujeto temático pueden repetirse y variarse, y sus resultados pueden conectarse y desconectarse de los términos operados. Como las ciencias naturales y formales son ciencias con un cierre flotante. Ahora bien, en las ciencias humanas y etológicas, la investigación científica supone, en mayor o menor medida, la modificación de las operaciones de los sujetos temáticos (hablantes, nativos, agentes sociales o económicos) de modo que la ciencia humana estricta presenta cierta continuidad con las técnicas de control de las operaciones, con las técnicas de control psicológico, de dominación colonial, de control social o económico, etc. (Alvargonzález 2013).

Las ciencias históricas, por su parte, son ciencias con un cierre fijo y la construcción de ese individuo único particular, cuya figura más clara son las instituciones y los imperios que intentan llevar a cabo proyectos de alcance universal, pone a estas ciencias en continuidad con la filosofía de la historia. Esto es así porque la conexión entre las partes de ese individuo global no se deduce exclusivamente de los propios materiales del campo científico, sino que implica movilizar principios ontológicos, antropológicos, de filosofía política, de filosofía de la religión, etc. que son los propios de la filosofía sistemática. En cualquier caso, en estas ciencias históricas no se aprecia esa conexión con las técnicas de control operatorio a las que antes me refería porque sobre los muertos no cabe control operatorio alguno. 


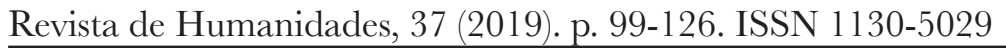

Considerando el grupo de las ciencias históricas resulta relevante distinguir entre la historia propiamente dicha y la arqueología y la prehistoria. Así como la presencia del lenguaje de palabras es uno de los criterios más firmes para establecer la diferencia entre las ciencias humanas propiamente dichas y las ciencias etológicas, la presencia de la escritura marca otra diferencia gnoseológica importante, la que existe entre, por un lado, la prehistoria y la arqueología que estudian sociedades ágrafas y monumentos sin escritura y, por otro lado, la historia propiamente dicha en cuyo campo hay textos escritos por humanos pretéritos.

Para terminar este párrafo quisiera hacer una breve referencia a la ciencia criminalística: a mi juicio, el proceso que conduce a los criminalistas a determinar los acontecimientos pasados (por tanto, ya cerrados) en torno a un suceso delictivo tiene la misma estructura gnoseológica que la historia fenoménica, esa historia que reconstruye eventos pasados. En ambos casos, se podrá hacer uso de tecnologías muy sofisticadas (por ejemplo, tecnologías de datación, o de identificación), pero la reconstrucción final de los hechos implica tomar en consideración las operaciones de los agentes implicados a la misma escala a la que éstas se produjeron. Claro está que, en la situación forense, a la reconstrucción de los hechos pretéritos le sigue la vista oral en la que defensa y acusación se co-determinarán mutuamente en una dialéctica que es la propia de las técnicas humanas de persuasión.

\subsection{La clasificación propuesta desde la teoría de los tipos de cierre}

La clasificación de las ciencias que acabo de proponer está basada en la especificidad de los términos y las operaciones materiales de cada uno de los grupos de ciencias en la medida en que afecta a los procesos de elaboración de las verdades científicas y a la consecución del cierre categorial de esas ciencias. Por lo que hace a este último asunto, se puede decir que esta clasificación recoge las modalidades más importantes de tener lugar el cierre categorial.

Es preciso distinguir las ciencias formales del resto de las ciencias porque en las ciencias formales, dado el carácter auto-referente de sus términos y operaciones, el cierre proposicional y el cierre objetual coinciden; esto no ocurre en las otras ciencias (naturales, humanas e históricas) en las que el estrato y el cierre objetual se distinguen del estrato y el cierre proposicional, y en las que el cierre operatorio objetual determina el proposicional.

La distinción de las ciencias históricas frente al resto de las ciencias, como ya he comentado, también se fundamenta en la diferenciación de dos modalidades del cierre categorial: los cierres fijos, propios de las ciencias históricas, y los cierres flotantes, propios del resto de las ciencias (formales, naturales y humanas). Los cierres fijos son una especie degenerada de cierres por el modo como quedan ancladas las operaciones en el campo. 
Por último, la distinción de las ciencias humanas y etológicas frente al resto de ciencias (formales, y naturales) tiene el siguiente fundamento: en las ciencias humanas y etológicas, incluso dando por supuesta la existencia de teoremas, la ausencia de principios de cierre o la coexistencia de principios incompatibles entre sí hacen que ese cierre campo sea problemático y no logre constituirse plenamente. Esto no ocurriría en las ciencias formales y naturales. Las ciencias históricas compartirían con las humanas la especificidad de un cierre anómalo, aunque por otras razones.

\section{LAS TREGE COMBINACIONES DE LOS CUATRO TIPOS BÁSICOS}

Como he comentado anteriormente, la clasificación gnoseológica de las ciencias que he propuesto procede por una doble vía: por vía ascendente, se parte de la multiplicidad de las ciencias modernas realmente existentes, y por vía descendente, se parte de una de las determinaciones constitutivas de toda ciencia, a saber: la presencia de operaciones en los campos científicos, y el modo cómo la naturaleza de las operaciones y de los términos operados afecta a la estructura gnoseológica de las ciencias y a su cientificidad.

Como toda teoría de naturaleza filosófica, esta propuesta debe ser puesta a prueba en la confrontación con otras clasificaciones alternativas. A continuación, voy a pasar revista brevemente a las trece posibilidades combinatorias que se pueden generar al agrupar entre sí los cuatro tipos de ciencias que he distinguido tomando como principio que todas las ciencias consideradas tienen que estar en la clasificación. Siete de estas posibilidades distinguen dos grupos de ciencias, ya sea agrupando tres de ellas frente a la cuarta (cuatro posibilidades) o ya sea agrupándolas de dos en dos (tres posibilidades); las otras seis posibilidades restantes, hasta completar las trece, dan lugar a clasificaciones de tres miembros.

\section{1. Ciencias formales frente a ciencias reales, experimentales o empíricas}

Ciencias formales / ciencias empíricas [naturales + humanas y etológicas + históricas]

Este modelo está actuando en la propuesta que hizo Wilhelm Wundt en su Sistema de filosofia científica (1889). La clasificación de Wundt, distingue tres tipos de ciencias, pero en realidad Wundt está utilizando dos clasificaciones basadas en criterios diferentes: la primera distingue entre ciencias de formas puras y ciencias experimentales o empíricas y utiliza un criterio epistemológico; la segunda, ya dentro de las ciencias empíricas distingue dos esferas ontológicas de la realidad, la Naturaleza y la Cultura. Podría interpretarse que la primera dicotomía (ciencias formales frente a ciencias reales o empíricas) es la que nos pone más cerca de la escala gnoseológica por cuanto supone diferenciar aquellas ciencias en las que el cierre proposicional y el cierre objetual coincide (las llamadas ciencias formales) de 


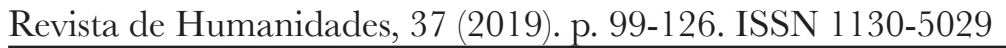

aquellas otras (el resto) en las que no se da esa coincidencia. Cuando comenté los criterios para diferenciar las ciencias formales del resto, en el apartado cuarto, ya hice un breve comentario a la propuesta de Wundt.

\subsection{Ciencias naturales frente al resto}

Ciencias naturales / ciencias no naturales [formales + humanas y etológicas + históricas]

La clasificación de las ciencias de Kedrov (1961) parte de que las ciencias propiamente dichas, las ciencias básicas o fundamentales, son las ciencias naturales porque son las que tratan directamente del mundo material y es ese mundo material el que determina el ser del hombre. En el sistema de las ciencias de Kedrov, las ciencias naturales se oponen a las ciencias humanas y este segundo grupo incluye las ciencias sociales y las ciencias que Kedrov llama "filosóficas", entre las que está la lógica. Según el acento que se ponga en unas ciencias u otras esta clasificación puede considerarse muy cercana a la que defendió Engels, y que comentaré más abajo (en el punto 8). Para el materialismo gnoseológico, las ciencias naturales son las únicas en las que tiene lugar un cierre categorial canónico: un cierre que no es exclusivamente proposicional y que no es fijo sino flotante.

\subsection{Ciencias humanas frente al resto}

Ciencias humanas / ciencias no humanas [formales + naturales + históricas]

Esta posibilidad combinatoria no da lugar a ninguna clasificación que haya sido defendida por ningún autor relevante. La clasificación de Ampère comentada más abajo (en el punto 5) podría considerarse bastante próxima si pudiéramos interpretar la historia en términos de historia natural. Para el materialismo gnoseológico esta clasificación recoge la distinción entre unas ciencias en las que hay un cierre efectivo (sea fijo, sea flotante, sea exclusivamente proposicional o no) frente a las ciencias humanas y etológicas en las que, ya sea por ausencia o por superabundancia de principios, no se da un cierre categorial efectivo y, a lo sumo, en el mejor de los casos, se logra un cierre técnico.

\subsection{Ciencias históricas frente a ciencias no históricas}

Ciencias históricas / ciencias no históricas [formales + naturales + humanas y etológicas]

En la doctrina de Domingo Báñez, Dios dispone de una ciencia de visión, por la cual conoce las cosas una vez han sucedido, y una ciencia de simple inteligencia, que le permite conocer todo lo posible. David Alvargonzález ha ensayado una interpretación según la cual la ciencia de visión se podría coordinar con la historia, 
y la ciencia de simple inteligencia cubriría el resto de las ciencias. Luis de Molina propuso la existencia de una tercera ciencia divina, la ciencia media, que permitiría a Dios conocer los futuros contingentes y que podría hacerse corresponder con las técnicas humanas efectivas (Alvargonzález 2013).

Bacon, en su De dignitate et augmentis scientiarum, adoptó como criterio de clasificación de las ciencias la teoría de las tres facultades del alma (imaginación, memoria, y razón), distinguió las ciencias de la memoria (la historia y sus variedades) y las ciencias de la razón (el resto de ciencias). A estos dos grupos se uniría el grupo de las ciencias de la imaginación (la poesía), pero éstas nos sacarían fuera del terreno de lo que hoy consideramos ciencias estrictas (Bacon 1623, lib. II). Ya he citado la filosofía de Giambattista Vico, que distinguió la historia del resto de las ciencias (Vico 1710 y 1725). Wilhelm Windelband, en el famoso discurso rectoral que pronunció en la Universidad de Estrasburgo en 1894, siguiendo la estela de Vico, también defendió la especificidad de la historia: las ciencias históricas, entendidas como ciencias de lo singular (idiográficas) son capaces de reconstruir las causas de los procesos pretéritos, mientras que el resto de las ciencias establecen leyes (ciencias nomotéticas) que no son más que funciones que ligan variables sin poder aclarar el significado de esas regularidades (Windelband 1894, Rickert 1922). Desde los presupuestos del materialismo filosófico esta clasificación recoge la diferencia entre cierres fijos (en la historia) y cierres flotantes (en el resto de las ciencias).

\subsection{Ciencias naturales frente a ciencias humanas y etológicas}

Ciencias naturales [formales + naturales] / ciencias humanas [humanas y etológicas + históricas]

Esta posibilidad es, sin duda, una de las más importantes y potentes de todas las que se pueden tomar en consideración. Los antecedentes podrían rastrearse en la distinción de Ibn Hazm de Córdoba entre, por un lado, las ciencias comunes a todos los pueblos, la aritmética, la medicina, la filosofía, y la astronomía y, por otro lado, las ciencias particulares de cada pueblo, el trívium que comprendía la religión, la historia y la lengua. Es necesario tener en cuenta que la clasificación de Ibn Hazm fue propuesta mucho antes de que existieran las ciencias en sentido moderno y, por tanto, mezcla saberes científicos y no científicos, además de tener una intención reivindicativa de las ciencias particulares de cada pueblo ligadas a la religión.

En un contexto histórico posterior a la existencia de las ciencias en sentido estricto, André-Marie Ampère clasificó las ciencias en dos grupos: las ciencias cosmológicas (matemáticas, física, ciencias naturales, ciencias médicas) y las ciencias noológicas (filosóficas, dialegmáticas, etnológicas y políticas) (Ampère 1834). También Wilhelm Dilthey, en su Introducción a las ciencias del espiritu, distinguió dos grupos de ciencias, las de la Naturaleza y las del Espíritu, pero es necesario precisar que esta dicotomía, lo mismo que en el caso de la propuesta de Ampère, descansaba en una ontología dualista (Dilthey 1883). 


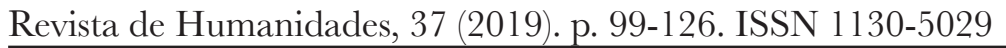

Gustavo Bueno, desde los presupuestos de la filosofía del cierre categorial, propuso una clasificación gnoseológica de las ciencias en dos grupos: naturales frente a humanas y etológicas. El criterio que he defendido unas líneas más arriba para distinguir estos dos grupos de ciencias (en el apartado 4.2) es exactamente el propuesto por Gustavo Bueno (1978).

\subsection{Ciencias antropogénicas frente al resto}

Ciencias productivas o antropogénicas [formales + históricas] / ciencias no productivas [naturales + humanas y etológicas]

Esta posibilidad combinatoria tendría la virtualidad de subrayar la proximidad de los campos de las ciencias formales con los de las ciencias históricas ya que en ambos casos esos campos están construidos con productos exclusivos de la acción humana: son campos de génesis antropológica, producidos ad hoc para esas ciencias. Frente a esas ciencias "productivas" se podrían situar el resto de las ciencias, naturales y humanas, si suponemos que estas últimas se desarrollan por procedimientos idénticos a las primeras. Algunas posiciones de los matemáticos intuicionistas podrían ser compatibles con este modo de entender las ciencias formales.

\section{6,7. Proximidad entre las ciencias formales y las ciencias humanas}

[cienciasformales + cienciashumanas] / [cienciasnaturales + cienciashistóricas]

Esta opción no ha sido defendida de un modo explícito, pero, aun así, tiene cierto interés teórico. En primer lugar, está ejercitada en todas aquellas historias globales que empiezan con la historia del cosmos y terminan con la historia política, cuando estas síntesis son presentadas como la ciencia evolutiva global. Por otra parte, esta posibilidad subrayaría la conexión de las ciencias formales con otras ciencias humanas entre las que se encuentra, sin duda, la psicología. Alexander Bain, en su crítica al sistema de las ciencias de Spencer, subrayó la conexión de la psicología con la lógica, una conexión que aparece también el sistema de las ciencias de Piaget pues ese sistema tiene la topología de un toro: en el uroboros piagetiano, el lugar en el que la serpiente se come la cola es precisamente el de la conexión entre las ciencias formales y la psicología (Piaget 1967).

\subsection{Ciencias formales, naturales y humanas.}

Ciencias formales / ciencias naturales / ciencias humanas [ciencias humanas y etológicas + ciencias históricas]

La clasificación de las ciencias en tres grupos, formales, naturales y humanas, es una de las opciones más transitadas a lo largo de la historia. Podríamos decir que, en un contexto ontológico metafísico, el primero que exploró esta posibilidad fue 
Hegel, en su Enciclopedia de las ciencias filosóficas, en donde distinguió la lógica, la filosofía de la naturaleza y la filosofía del espíritu (Hegel 1817). Engels, aun desde presupuestos contrarios al idealismo hegeliano, respetó esta tripartición, en su Dialéctica de la Naturaleza, al distinguir las ciencias naturales, las ciencias humanas, y las ciencias del pensamiento, que se corresponderían con la lógica (Engels 1876). En la clasificación de las ciencias de Wilhelm Wundt también se mantuvo esa trinidad por medio de una doble clasificación: en primer lugar, la distinción entre ciencias formales y ciencias empíricas o reales y, en segundo lugar, la división de las ciencias empíricas en ciencias naturales y ciencias del espíritu de acuerdo con sus respectivos objetos de estudio (Wundt 1889). Wilhelm Ostwald también mantuvo en lo esencial la estructura de la clasificación hegeliana acuñando nuevas denominaciones: en las ciencias del orden incluyó la lógica, las matemáticas y la mecánica, en las ciencias energéticas agrupó la física, la química, y la fisiología y, por último, en las que llamó ciencias "biológicas" incluyo las que, para nosotros, son ciencias etológicas y humanas, la psicología, la culturología y la sociología (Ostwald 1914).

\subsection{Ciencias formales, ciencias históricas y el resto}

Ciencias formales / ciencias históricas / ciencias predictivas [naturales + ciencias humanas y etológicas].

Esta opción sería compatible con una filosofía de la ciencia que, por una parte, considere a las ciencias formales como un instrumento o un lenguaje puesto al servicio de las otras ciencias y que, por otra parte, sea escéptica acerca de la cientificidad de la historia, de modo que las ciencias propiamente dichas serían las ciencias empíricas, predictivas, no históricas. La distinción de primer Popper entre unas ciencias formales, unas ciencias de explicación de acontecimientos ya ocurridos, y unas ciencias predictivas, podría aproximarse bastante a este modelo (Popper 1934).

6.10. Ciencias estrictas (formales y naturales), ciencias humanas y ciencias históricas

Ciencias estrictas [naturales + formales] / ciencias humanas / ciencias históricas

La posibilidad de reunir en un mismo grupo las ciencias naturales y las ciencias formales es muy tentadora ya que en ambos casos estamos hablando de ciencias modernas en sentido estricto, de ciencias en las que la verdad de sus teoremas es universalmente admitida. La propuesta defendida en este artículo, por tanto, se aproxima mucho a esta opción ya que en ningún momento se pone en duda la cientificidad estricta de estas disciplinas. Como he argumentado (en el apartado 4.1) el carácter auto-referente de los términos de las ciencias formales sólo afecta 


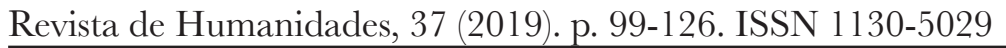

a la imposibilidad de construir un único cierre categorial que englobe a todas las matemáticas (o lógicas) coordinadas por unos mismos principios, pero no afecta para nada a la cientificidad de los teoremas matemáticos (y lógicos) ni a la cientificidad de cada una de las partes de las matemáticas (y de la lógica). La lógica y las matemáticas se caracterizarían por su manera sui generis (pragmática) de asignar contenidos semánticos a sus términos, relaciones y operaciones, pero en todo lo demás pueden considerarse como el canon de las ciencias estrictas y, por tanto, están estrechamente emparentadas con las ciencias naturales. Además, en los campos de las ciencias formales no aparece el sujeto temático lo cual marca una diferencia muy importante con las ciencias humanas, etológicas e históricas.

6.11. Ciencias productivas, ciencias naturales y ciencias humanas

Ciencias productivas [formales + históricas] / ciencias naturales / ciencias humanas

Como ya hemos visto en la sexta posibilidad combinatoria, la doctrina de Vico (1710 y 1725) del verum est factum, con los antecedents citados de Hobbes (1658), permite acercar gnoseológicamente las ciencias formales a las ciencias históricas. Luego quedarían las ciencias que no dependen ya tan directamente de las acciones de los hombres que, a su vez, se podrían distinguir, incluso desde el espacio antropológico plano (radial + circular) propio de la filosofía cristiana.

\subsection{Ciencias formales, ciencias humanas y el resto}

Ciencias en perspectiva histórica [historia natural + historia civil] / ciencias formales / ciencias humanas.

Esta posibilidad combinatoria pertenece a la misma familia que la anteriormente expuesta en el número siete. Las disciplinas históricas, tanto de la historia natural como de la historia civil, van juntas. Luego quedan las ciencias formales como una especie de organon o ciencia instrumental y las disciplinas humanas y etológicas que plantearían problemas especiales desde una perspectiva, por ejemplo, emergentista.

\subsection{Ciencias naturales, ciencias históricas y el resto}

[ciencias formales + ciencias humanas] / ciencias naturales / ciencias históricas

Esta posibilidad combinatoria también nos remite a lo dicho en la opción séptima. Aquí la conexión se establece entre las ciencias formales y ciertas ciencias 
humanas y etológicas. Citábamos entonces a Bain y a Piaget. Claro que ellos no defendieron la separación de las ciencias históricas frente al resto de las ciencias humanas ya que su perspectiva era más bien psicosociológica.

\section{CONTRIBUGIONES DE ESTA PROPUESTA}

En este artículo he propuesto un criterio para clasificar las ciencias modernas: atenerse a la naturaleza de los términos operados por el científico y por el sujeto temático. Las contribuciones más relevantes de esta propuesta, si se compara con la teoría de Bueno (1993, §36), son las siguientes:

1. De este modo se logra unificar en una misma clasificación los grupos de las ciencias formales, las ciencias naturales, las ciencias humanas con sus variedades, y las ciencias históricas. Algunos agrupamientos de estos cuatro tipos permiten dar cuenta de las clasificaciones de las ciencias más relevantes.

2. Se argumenta que la distinción entre ciencias formales y ciencias no formales es gnoseológica porque:

2.1. Permite dar cuenta de las razones internas por las que existe una multiplicidad de disciplinas científicas lógicas y matemáticas, y por qué esta anomalía no se da en el resto de las ciencias.

2.2. Permite entender mejor el modo sui generis de establecer los contenidos de estas ciencias, y las razones por las que la doctrina de la verdad por coherencia resulta fértil en sus campos.

3. Se incorpora de manera interna la demarcación entre lógica y matemáticas atendiendo a la naturaleza de las operaciones (autoformantes y heteroformantes).

4. Al tomar en consideración la naturaleza de los términos operados por los sujetos temáticos se pueden distinguir las ciencias etológicas de las propiamente humanas.

5. Se argumenta que la distinción entre ciencias históricas y el resto de las ciencias no históricas tiene significado gnoseológico porque:

5.1 La ausencia del sujeto temático en el campo de las ciencias históricas compromete el modo de ligar sus operaciones en cierres fijos, mientras que en el resto de las ciencias la posibilidad de repetir las operaciones deja los resultados de éstas libres, "flotando" para ulteriores procesos operatorios.

5.2. Como las ciencias históricas reconstruyen operaciones pretéritas humanas, se entiende por qué la doctrina de la verdad científica como adecuación es especialmente fértil en sus campos

5.3. Por la misma razón, la distinción entre las ciencias históricas y el resto de las ciencias humanas permite entender internamente la conexión de las primeras con 


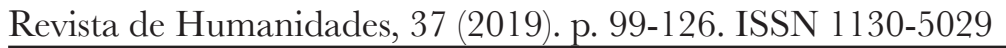

la filosofía de la historia y de las segundas con las técnicas efectivas de control de las operaciones animales o humanas.

\section{BIBLIOGRAFÍA CITADA}

Alvargonzález, D. (1989) Problemas en torno al concepto de 'ciencias humanas' como ciencias con doble plano operatorio. El Basilisco 2 (1989): 51-56.

Alvargonzález, D. (1994) Análisis gnoseológico del campo de la teoría de juegos. El Basilisco 28 (2000): 17-36.

Alvargonzález, D. (2011). Multidisciplinarity, interdisciplinarity, transdisciplinarity, and the sciences. International Studies in the Philosophy of Science 25/4: 387-403.

Alvargonzález, D. (2013). Ciencias humanas y ciencias divinas. Daimon. Revista Internacional de Filosofía 58: 109-124.

Ampère, A-M. (1834). Essai sur la philosophie des sciences ou expositon analytique d'une classification naturelle de toutes les connaissances humaines. París, Francia: Bachelier.

Bacon, R. (1267). Opus Majus [Gran obra]. Frankfurt Main, Alemania: 1964.

Brockman, J. (Ed.). (1995). La tercera cultura. Más allá de la revolución científica. Barcelona: Tusquets, 1996.

Bueno, G. (1970). El papel de la filosofía en el conjunto del saber. Madrid, España: Ciencia Nueva.

Bueno, G. (1976.). Estatuto gnoseológico de las ciencias humanas. Madrid, España: Fundación Juan March.

Bueno, G. (1978). En torno al concepto de ciencias humanas. El Basilisco 2: 12-46.

Bueno, G. (1978). Operaciones autoformantes y heteroformantes. Ensayo de un criterio de demarcación gnoseológica entre la Lógica formal y la Matemática (I) El Basilisco 7:16-39.

Bueno, G. (1979). Operaciones autoformantes y heteroformantes. Ensayo de un criterio de demarcación gnoseológica entre la Lógica formal y la Matemática (II) $\mathrm{El}$ Basilisco 8:4-25.

Bueno, G. (1980). El individuo en la historia. Oviedo, España: Universidad de Oviedo.

Bueno, G. (1989) Sobre el alcance de una 'ciencia media' (ciencia $\beta_{1}$ ) entre las ciencias humanas estrictas (ciencias $\alpha_{2}$ ) y los saberes prácticos positivos $\left(\beta_{2}\right)$. El Basilisco 2 (1989): $57-73$

Bueno, G. (1993). Teoría del cierre categorial. Oviedo, España: Pentalfa.

Bueno, G. (1995a). ¿Qué es filosofia? Oviedo, España: Pentalfa.

Bueno, G. (1995b) Scala Naturae. Ciclo de conferencias en el Departamento de Filosofía de la Universidad de Oviedo (14 y 21 de marzo y 4 de abril). 
La clasificación de las ciencias desde la filosofía... - David Alvargonzález

Bueno, G. (1995c). ¿Qué es la ciencia? Oviedo, España: Pentalfa.

Bueno, G. (2000a). Las matemáticas como disciplina científica. Ábaco 25-26: 48-71.

Bueno, G. (2000b). Televisión, apariencia y verdad. Barcelona: Gedisa.

Bueno, G. (2004). Propuesta de clasificación de las disciplinas filosóficas. El Catoblepas 28: 2.

Bunge, M. (1969). La investigación científica. Barcelona, España: Ariel.

Cartwright, N. (1999). The Dappled World: A Study of the Boundaries of Sciences. Cambridge, U.K.: Cambridge University Press.

Dilthey, W. (1883). Introducción a las ciencias del espiritu. Madrid, España: Alianza, 1956.

Dupré, J. (1993). The Disorder of Things. Cambridge MA, EUA: Harvard University Press.

Dupré, J. (1996). Metaphysical disorder and scientific disunity. En P. Galison y D.J. Stump (Ed.), The Disunity of Science. California, EUA: Stanford University Press.

Engels, F. (1876). Dialéctica de la Naturaleza. Méjico: Grijalbo, 1961.

Galison, P. and D. Stump (Ed.). (1996). The Disunity of Science. Boundaries, Contexts and Power. Stanford, EUA: Stanford University Press.

Hacking, I. (1996).The disunities of sciences. En P. Galison, y D.J. Stump (Ed.), The Disunity of Science. California, EUA: Stanford University Press.

Hegel, G.W.F. (1817). Enciclopedia de las ciencias filosóficas. Madrid, España: Alianza, 1997.

Hobbes, Th. (1658) Elementorum philosophiae sectio secunda De Homine. Edición de las obras latinas de William Molesworth, Londres: J. Bohn 1839-45, $2^{\circ}$ reimpresión: Aalen: Scientia Verlag, 1966, vol 2: 93.

Lalande, A. (1893). Lectures sur la philosophie des sciences. Paris, Francia: Hachette.

Mondolfo, R. (1969). Verum factum: desde antes de Vico hasta Marx. México: Siglo XXI, 1971.

Neurath, O., Carnap, R. and Morris, Ch. W (Ed.). (1938). Foundations of the Unity of Science: Toward an International Encyclopedia of Unified Science. Chicago, EUA: University of Chicago Press.

Ostwald, W. (1914). Moderne Naturphilosophie. Leipzig, Alemania: Akademische Verlagsgesellschaft.

Piaget, J. (1967). El sistema y la clasificación de las ciencias. En J. Piaget (Ed.) La lógica y el conocimiento científico. Barcelona, España: Paidós, 1979.

Popper, K.R. (1934). La lógica de la investigación científica, Madrid, España: Tecnos, 1985. 
Revista de Humanidades, 37 (2019). p. 99-126. ISSN 1130-5029

Rickert, H. (1922). Ciencia cultural ciencia natural. Madrid, España: Espasa Calpe, 1922.

Simmel, G. (1920). Die probleme der Gechichtsphilosophie. Eine erkentnistheoretische Studie. Munich, Alemania: Verlag von Dunker und Humboldt.

Simpson, G.G. (1964). This view of Life: The World of an Evolutionist. New York, EUA: Harcourt, Brace \& World.

Spencer, H. (1864). The Classification of the Sciences to which are added Reason for Dissenting from the Philosophy of M. Comte. London, UK: Williams and Norgate.

Vico, G. (1710). De antiquissima italorum sapientia ex linguae latinae originibus eruenda. http://www.giambattistavico.it/opere/deantiquissima

Vico, G. (1725). La ciencia nueva. Madrid, España: Tecnos, 2006.

Wilson, E. O. (1998). Consilience: La unidad del conocimiento. Barcelona, España: Galaxia Gutemberg, 1999.

Windelband, W. (1894). Historia y ciencia de la naturaleza. Discurso rectoral de la Universidad de Estrasburgo. En W. Windelband, Preludios filosóficos, Buenos Aires, Argentina: Santiago Rueda Editor, 1949.

Wundt, W.M. (1889). Sistema de filosofía cientifica. Fundamentos de metafísica basada en las ciencias positivas. Madrid, España: Daniel Jorro, 1913. 
